\title{
Global geometry of planary 3-body motions
}

\author{
Wu-Yi Hsiang \\ Department of Mathematics \\ University of California, Berkeley \\ Eldar Straume \\ Department of Mathematical Sciences \\ Norwegian University of Science and \\ Technology, Trondheim, Norway
}

September 26, 2018

\section{Contents}

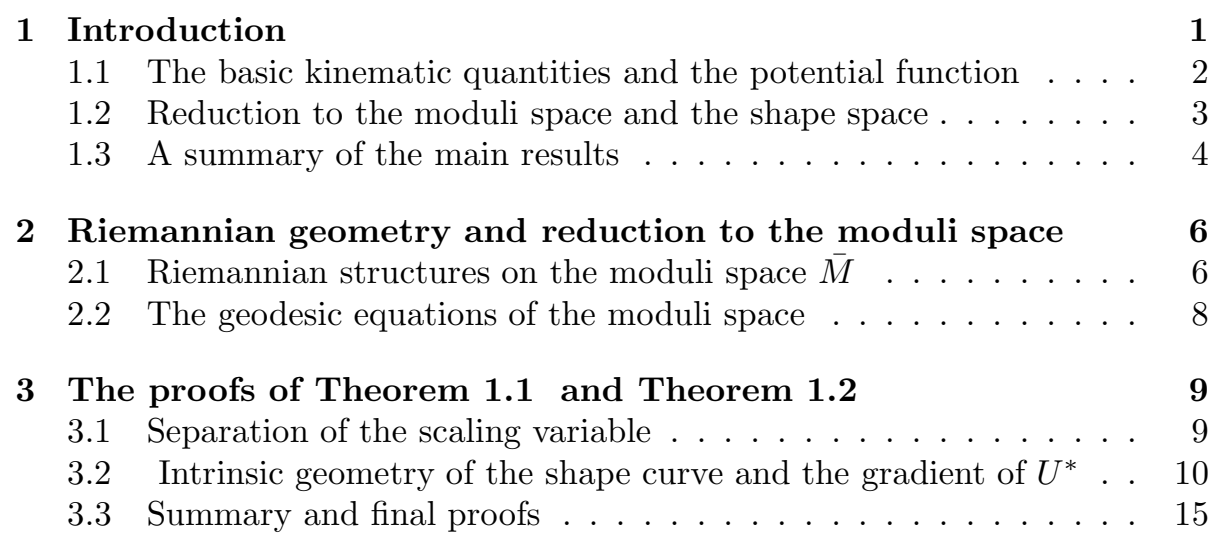

\section{Introduction}

In a recent paper (cf. 3]) the authors have investigated 3-body motions with vanishing angular momentum, in the framework of equivariant Riemannian geometry and by resuming the basic approach dating back to Jacobi's geometrization of Lagrange's least action principle, in the setting of kinematic geometry of 3-body systems. A geometric reduction method was described which reduces the study of trajectories of 3-body motions, first from the level of the configuration space to the level of the moduli space of congruence classes of m-triangles, and then 
- further reduces the moduli curves to that of their shape curves on the 2 -sphere $S^{2}$. Namely, a trajectory of 3-body motions is completely determined, up to global congruence, by its shape curve which only records the changing of shape (i.e. similarity class).

- Moreover, the unique parametrization theorem further proves that the trajectory is already determined by the geometric (i.e. non-parametrized) shape curve.

- Another remarkable property of the above shape curves is expressed by the monotonicity theorem, concerning their (mass modified) latitude function on the sphere $S^{2}$.

The monotonicity theorem is definitely only valid in the case of zero angular momentum, but with this paper we shall extend the first two of the above three stated results to the more general case of planary motions. We start with a description of the basic setting from [3] in the two subsections below, and a summary of the major results is presented in Section 1.3.

In Section 2 we work out the kinematic and dynamical metric on the moduli space, together with the associated differential equations. Finally, in Section 3 we establish the remaining results needed for the proofs of the two main theorems stated in Section 1.3.

\subsection{The basic kinematic quantities and the potential func- tion}

The classical 3-body problem in celestial mechanics studies the local and global geometry of the trajectories of a 3-body system, namely the motion of three point masses (bodies) of mass $m_{i}>0$, say normalized to $\sum m_{i}=1$, under the influence of the mutual gravitational forces. This system constitutes a conservative mechanical system with the Newton's potential function

$$
U=\sum_{i<j} \frac{m_{i} m_{j}}{r_{i j}}, \quad r_{i j}=\left|\mathbf{a}_{i}-\mathbf{a}_{j}\right|
$$

and potential energy $-U$. We introduce the vector $\delta=\left(\mathbf{a}_{1}, \mathbf{a}_{2}, \mathbf{a}_{3}\right)$, called an $m$-triangle, which records the position of the system in an inertial frame with the origin at the center of mass, and hence $\sum m_{i} \mathbf{a}_{i}=0$.

A trajectory is a time parametrized curve $\gamma(t)$ representing a motion of the 3-body system, locally characterized by Newton's equation

$$
\frac{d^{2}}{d t^{2}} \gamma=\nabla U(\gamma)=\left(\frac{1}{m_{1}} \frac{\partial U}{\partial \mathbf{a}_{1}}, \frac{1}{m_{2}} \frac{\partial U}{\partial \mathbf{a}_{2}}, \frac{1}{m_{3}} \frac{\partial U}{\partial \mathbf{a}_{3}}\right)
$$

However, the trajectories can also be characterized globally as solutions of a suitable boundary value problem, characterized as extremals of an appropriate least action principle, such as the two principles due to Lagrange and Hamilton. 
Let us also recall the basic kinematic quantities which are the (polar) moment of inertia, kinetic energy and angular momentum, respectively defined by

$$
I=\sum m_{i}\left|\mathbf{a}_{i}\right|^{2}, \quad T=\frac{1}{2} \sum m_{i}\left|\dot{\mathbf{a}}_{i}\right|^{2}, \quad \boldsymbol{\Omega}=\sum m_{i}\left(\mathbf{a}_{i} \times \dot{\mathbf{a}}_{i}\right)
$$

The dynamics of the 3-body problem is largely expressed by their interactions with the potential function $U$ via the equation (2), and for example, the invariance of the total energy

$$
h=T-U
$$

is a simple consequence of (2) and the definition of $T$. On the other hand, whereas the invariance of the vector $\boldsymbol{\Omega}$ follows from the rotational symmetry of $U$, in this article we shall exploit the consequences of the additional homogeneity property of $U$.

\subsection{Reduction to the moduli space and the shape space}

In this article we shall only be concerned with planary three-body motions, namely the m-triangles $\delta$ are confined to a fixed plane $\mathbb{R}^{2}$ and hence belong to the configuration space

$$
M \simeq \mathbb{R}^{4}: \sum_{i=1}^{3} m_{i} \mathbf{a}_{i}=0, \quad \mathbf{a}_{i} \in \mathbb{R}^{2}
$$

With the inner product of m-triangles defined by

$$
\delta \cdot \delta^{\prime}=\sum m_{i} \mathbf{a}_{i} \cdot \mathbf{b}_{i}
$$

$M$ is given the kinematic metric, namely the metric such that the right side of Newton's equation (2) is the gradient of $U$. Then the squared norm is the moment of inertia, $I=I(\delta)=|\delta|^{2}$, and hence the hyperradius $\rho=\sqrt{I}$ is the natural scaling function which also measures the distance from the origin.

The rotation group $S O(2)$ acts naturally, and by orthogonal transformations, on $M$ by rotating $\mathrm{m}$-triangles, and the orbit spaces of $M$ (resp. its unit sphere $M^{1} \simeq S^{3}$ ) are the (congruence) moduli space $\bar{M}$ (resp. the shape space $M^{*}$ ), namely

$$
\bar{M}=M / S O(2), \quad M^{*}=M^{1} / S O(2)
$$

The points in $\bar{M}$ represent congruence classes $\bar{\delta}$ of m-triangles, and points in $M^{*}$ represent the shapes (or similarity classes) $\delta^{*}$ of non-zero m-triangles.

Geometrically,the above orbit space construction and orbit map $M \rightarrow \bar{M}$ is, in fact, just the Hopf map construction, whose restriction $S^{3} \rightarrow S^{2}$ is the classical Hopf fibration. Namely, the spaces fit into the following diagram

$$
\begin{array}{ccc}
M \simeq & \mathbb{R}^{4} & \longrightarrow \bar{M} \\
\cup & \cup & \cup \\
M^{1} \simeq S^{3} & \rightarrow M^{*} \simeq S^{2}(1 / 2)
\end{array}
$$


where $M \simeq \mathbb{R}^{4}$ is a chosen $S O(2)$-equivariant isometry (i.e. choice of Jacobi vectors). In particular, $\bar{M} \approx \mathbb{R}^{3}$ is a cone over $M^{*}$ and there is the radial projection $\bar{M}-\{O\} \rightarrow M^{*}$ which "reduces" a non-zero congruence class $\bar{\delta}$ to its shape $\delta^{*}$. Note, however, the representation of the various shapes of $\mathrm{m}$-triangles on a fixed model sphere $S^{2}$ depends on the mass distribution $\left\{m_{i}\right\}$, via the mass dependence of the Jacobi vectors.

Briefly, in this article we shall analyze the two-step reduction

$$
M \rightarrow \bar{M}, \quad \bar{M}-\{O\} \rightarrow M^{*}, \quad \gamma(t) \rightarrow \bar{\gamma}(t) \rightarrow \gamma^{*}(t)
$$

by which a trajectory $\gamma(t)$ of a planary 3-body motion is projected to its moduli curve $\bar{\gamma}(t)$ and further to its shape curve $\gamma^{*}(t)$ on a 2-sphere. In Section 2.1 we shall put the above reduction and the spaces involved in the framework of Riemannian geometry, and moreover, explain how Jacobi's geometrization idea can be reduced and extended to the level of $\bar{M}$.

\subsection{A summary of the main results}

The Hopf map construction (8) makes it convenient to use a Euclidean model, $\bar{M}=\mathbb{R}^{3}$, for the moduli space and with the unit sphere $S^{2}(1)$ as the shape space $M^{*}$. In this way one can express all kinematic quantities and dynamical equations in terms of spherical geometry and spherical coordinates, and hence take the full advantage of the cone structure of $\bar{M}$ over $M^{*}$.

One can start from Newton's equation (2) for planary m-triangles

$$
\frac{d^{2}}{d t^{2}} \gamma=\nabla U(\gamma), \quad \gamma(t) \in M
$$

with any $S O(2)$-invariant potential function $U$, and hence it is a function on $\bar{M}$. The additional crucial property of $U$ that we have exploited is its homogeneity, namely it is of type

$$
U=\frac{U^{*}(\varphi, \theta)}{\rho^{e}}
$$

where $U^{*}$ denotes the restriction of $U$ to the sphere $M^{*}$. The Newtonian case $e=1$ is certainly the most important one, but the proofs are essentially the same for other (integral) values of $e>0$.

Consider trajectories $\gamma(t)$ of (10) for a given energy-momentum level $(h, \omega)$, $\omega=|\Omega|$, and for spherical coordinates $(\rho, \varphi, \theta)$ in $\bar{M}$, let the curves

$$
\bar{\gamma}(t)=\left(\rho(t), \gamma^{*}(t)\right), \quad \gamma^{*}(t)=(\varphi(t), \theta(t))
$$

be the associated moduli and shape curve, respectively. In Section 2.2 we show the reduced Newton's equation in $\bar{M}$ can be presented as the pair

$$
\ddot{I}=2(U+2 h)+2(1-e) U, \quad \ddot{\gamma}^{*}+P \dot{\gamma}^{*}+Q \nabla U^{*}=0
$$

where the first equation in (13) is simply the Lagrange-Jacobi equation, and $\ddot{\gamma}^{*}$ is the covariant acceleration of $\gamma^{*}$ as a spherical curve. Moreover, the energy 
integral (4) is the following first order equation in $\bar{M}$

$$
\frac{1}{2} \dot{\rho}^{2}+\frac{\rho^{2}}{8} v^{2}+\frac{\omega^{2}}{2 \rho^{2}}-\frac{U^{*}}{\rho^{e}}-h=0
$$

where $v=\left|\dot{\gamma}^{*}\right|=\sqrt{\dot{\varphi}^{2}+\left(\sin ^{2} \varphi\right) \dot{\theta}^{2}}$ is the speed of the shape curve. In fact, combined with (14) any of the three scalar equations in (13) can be derived from the other ones. The equations of (13) are presented in the coordinates $(\rho, \varphi, \theta)$ in Section 2.2.

On the other hand, let $K^{*}$ be the geodesic curvature of $\gamma^{*}$ and $U_{\nu}^{*}$ the directional derivative of $U^{*}$ normal to $\gamma^{*}$. Then there is the formula

$$
\rho^{2+e}=\frac{4}{v^{2}} \mathfrak{S}, \quad \text { where } \mathfrak{S}=\frac{U_{\nu}^{*}}{K^{*}} \quad(\text { cf. } \quad \text { (37) })
$$

which separates the radial variable $\rho$ from the spherical ones. Using (15), the dependence on $\rho$ in the coefficient functions $P, Q$ in (13) can be eliminated, namely

$$
P=2 \frac{\dot{\rho}}{\rho}=\frac{2}{2+e} \frac{\dot{\mathfrak{S}}}{\mathfrak{S}}-\frac{4}{2+e} \frac{\dot{v}}{v}, \quad Q=-\frac{4}{\rho^{2+e}}=-\frac{v^{2}}{\mathfrak{S}},
$$

which yields a third order equation for $\gamma^{*}$ which is, in fact, independent of $(h, \omega)$.

Clearly, the above function $\mathfrak{S}$ depends only on the relative geometry between $\gamma^{*}$ and the gradient of $U^{*}$. However, we regard $\mathfrak{S}$ as undefined if $\gamma^{*}$ is a geodesic arc (and hence lies on a gradient line), and any such solution of (13) is called exceptional. Now, assuming (for simplicity) that the shape curve is not of exceptional type, our main results can be formulated neatly as the following two theorems :

Theorem 1.1 For a given total energy and nonzero angular momentum, a planary three-body motion is completely determined up to congruence by its time parametrized shape curve (which only records the changing of shape).

Theorem 1.2 The time parametrization is uniquely determined by the relative geometry between the oriented geometric (i.e. non-parametrized) shape curve and the gradient vector field of $U^{*}$.

Remark 1.3 (i) In the case of non-zero total energy, Theorem 1.1 remains unchanged in the case of zero angular momentum, whereas the motion is determined up to congruence and scaling in the case of $(h, \omega)=(0,0)$. We refer to [3], Section 4.2.

(ii) Uniqueness of time parametrization means, of course, modulo time translation, or modulo an affine time transformation when $(h, \omega)=(0,0)$.

(iii) The proofs of the above theorems are the same for any homogeneous potential function of type [11), with $e>0$, and e integral in Theorem 1.2. We choose the most important case, $e=1$, in Section 3.2 and work out the crucial details. However, the formulae are even simpler in the case $e=2$. 


\section{Riemannian geometry and reduction to the moduli space}

\subsection{Riemannian structures on the moduli space $\bar{M}$}

In his famous lectures 4], Jacobi introduced the concept of a kinematic metric $d s^{2}$ on the configuration space $M$ of a mechanical system with kinetic energy $T$. For example, in the case of an n-body system with total mass $\sum m_{i}=1$,

$$
d s^{2}=2 T d t^{2}=\sum_{i} m_{i}\left(d x_{i}^{2}+d y_{i}^{2}+d z_{i}^{2}\right)
$$

which is clearly equivalent to the definition (6). Now, for a system with potential energy $-U$ and a fixed total energy $h$, set

$$
\begin{aligned}
& M_{h}=\{p \in M ; h+U(p) \geq 0\} \\
& d s_{h}^{2}=(h+U) d s^{2}
\end{aligned}
$$

where $d s_{h}^{2}$ is called the dynamical metric on $M_{h}$. By writing

$$
d s_{h}=\sqrt{h+U} d s=\sqrt{T} d s=\sqrt{2} T d t
$$

Jacobi transformed Lagrange's action integral (on the left side of (19)) into an arc-length integral, namely

$$
J(\gamma)=\int_{\gamma} T d t=\frac{1}{\sqrt{2}} \int_{\gamma} d s_{h}
$$

and hence the least action principle becomes the following simple geometric statement :

" Trajectories with total energy $h$ are exactly those geodesic curves

in the space $M_{h}$ with the dynamical metric $d s_{h}^{2} "$

Nowadays, the metric spaces $\left(M, d s^{2}\right),\left(M_{h}, d s_{h}^{2}\right)$ are called Riemannian manifolds, and the dynamical metric is a conformal modification of the kinematic metric by the scaling function $(U+h)$. As exemplified by (17), a Riemannian metric on a manifold $N$ amounts to the choice of a kinetic energy function on the tangent bundle, $T: T N \rightarrow \mathbb{R}$, which is a positive definite quadratic form on each tangent plane $T_{p} N$. This allows us to define the arc-length function $u(t)$ and the kinetic energy along a given time parametrized curve $\Gamma(t)$ by

$$
T(t)=\frac{1}{2}\left(\frac{d u}{d t}\right)^{2}=\frac{1}{2}\left|\frac{d \Gamma}{d t}\right|^{2}
$$

Now, let us determine the appropriate kinetic energy $\bar{T}$ on the moduli space $\bar{M}$ and hence also define its kinematic metric using the recipe (21). At the 
same time, referring to the diagram (8) and the Hopf map, let us also introduce the orbital distance metric $d \bar{s}^{2}$ on $\bar{M}$ as an $S O(2)$-orbit space. Then $\left(\bar{M}, d \bar{s}^{2}\right)$ inherits the structure of a Riemannian cone over the shape space $\left(M^{*}, d \sigma^{2}\right)$, namely

$$
d \bar{s}^{2}=d \rho^{2}+\rho^{2} d \sigma^{2}, \quad d \sigma^{2}=\left.d \bar{s}^{2}\right|_{M^{*}}
$$

Moreover, it is well known that the Hopf fibration in the above Riemannian setting is $S^{3}(1) \rightarrow S^{2}(1 / 2)$, and consequently

$$
\left(M^{*}, d \sigma^{2}\right) \simeq S^{2}(1 / 2)
$$

is also the round sphere of radius $1 / 2$.

Consider a curve $\gamma(t)$ in $M$ and its orthogonal velocity decomposition $\dot{\gamma}=$ $\dot{\gamma}^{h}+\dot{\gamma}^{\omega}$ and corresponding splitting of kinetric energy

$$
T=T^{h}+T^{\omega},
$$

where $\dot{\gamma}^{\omega}$ is tangential to the $S O(2)$-orbit, and hence $T^{\omega}$ is the kinetic energy due to purely rotational motion of m-triangles. By definition of the metric $d \bar{s}^{2}$, the orbit map $M \rightarrow \bar{M}$ is a Riemannian submersion and hence maps the "horizontal" component $\dot{\gamma}^{h}$ isometrically to the velocity vector of $\bar{\gamma}$. This shows $T^{h}=\bar{T}$ is also the kinetic energy at the level of $\bar{M}$, that is, the kinematic metric coincides with the orbital distance metric, and by (22), (23) and (24) the latter can be finally expressed as

$$
\begin{aligned}
d \bar{s}^{2} & =2 \bar{T} d t^{2}=2\left(T-T^{\omega}\right) d t^{2}=2\left(T-\frac{\omega^{2}}{2 \rho^{2}}\right) d t^{2} \\
& =d \rho^{2}+\rho^{2} d \sigma^{2}=d \rho^{2}+\frac{\rho^{2}}{4}\left(d \varphi^{2}+\sin ^{2} \varphi d \theta^{2}\right)
\end{aligned}
$$

Remark 2.1 The expression $d \varphi^{2}+\sin ^{2} \varphi d \theta^{2}$ in the last line of 25) is the metric of the unit sphere $S^{2}(1)$ in terms of spherical polar coordinates. In fact, the metric $d \bar{s}^{2}$ differs from the Euclidean metric only by the factor $1 / 4$ in (25), which makes it singular at the origin. Moreover, $d \bar{s}^{2}$ is actually a conformal modification of the Euclidean metric (cf. [3], Section 2).

Next, we turn to the construction of the dynamical metric on the moduli space $\bar{M}$, which depends on $U$ and a given energy-momentum level $(h, \omega)$. Following the geometrization idea of Jacobi, we want the geodesics of this metric to be the trajectories in $\bar{M}$, regarded as a simple mechanical system with kinetic energy $\bar{T}$, potential energy $\bar{U}$, and conserved total energy $h=\bar{T}-\bar{U}$. Thus we introduce the reduced potential function on $\bar{M}$

$$
\bar{U}=U-\frac{\omega^{2}}{2 \rho^{2}}
$$

and define the dynamical metric

$$
d \bar{s}_{h, \omega}^{2}=\bar{T} d \bar{s}^{2}=(\bar{U}+h) d \bar{s}^{2}=\left(U+h-\frac{\omega^{2}}{2 \rho^{2}}\right) d \bar{s}^{2}
$$


Finally, it is not difficult to see that Lagrange's least action principle (19) as well as Hamilton's least action principle using the Lagrange function $L=T+U$, can be pushed down to the level of $\bar{M}$. This yields the function $\bar{L}=\bar{T}+\bar{U}$, and for example, by following Jacobi's geometrization idea applied to Lagrange's action integral in $\bar{M}$

$$
\bar{J}(\bar{\gamma})=\sqrt{2} \int_{\bar{\gamma}} \bar{T} d t=\sqrt{2} \int_{\bar{\gamma}}(\bar{U}+h) d t=\int_{\bar{\gamma}} \sqrt{\bar{U}+h} d \bar{s}=\int d \bar{s}_{h, \omega},
$$

we arrive at the following geometric statement similar to (20):

"Curves in $\bar{M}$ representing trajectories in $M$ at a given energy-momentum level $(h, \omega)$ are exactly those geodesic

$$
\text { curves in } \bar{M} \text { with the induced dynamical metric } d \bar{s}_{h, \omega}^{2} . "
$$

\subsection{The geodesic equations of the moduli space}

The moduli space $\bar{M}$ is, first of all, equipped with the kinematic metric

$$
d \bar{s}^{2}=2 \bar{T} d t^{2}=2\left(T-\frac{\omega^{2}}{2 \rho^{2}}\right) d t^{2}=d \rho^{2}+\frac{\rho^{2}}{4}\left(d \varphi^{2}+\sin ^{2} \varphi d \theta^{2}\right)
$$

and for each energy-momentum level $(h, \omega)$ there is the following conformal modification of $d \bar{s}^{2}$

$$
d \bar{s}_{(h, \omega)}^{2}=\bar{T} d \bar{s}^{2}=(\bar{U}+h) d \bar{s}^{2}
$$

The latter is the dynamical metric which characterizes those moduli curves $\bar{\gamma}(t)$ representing trajectories $\gamma(t)$ at the specified level $(h, \omega)$. Namely, $\bar{\gamma}$ is a geodesic of the Riemannian metric (29), which in the spherical coordinates $(\rho, \varphi, \theta)$ expresses as

$$
d \bar{s}_{(h, \omega)}^{2}=\left(\frac{U^{*}(\varphi, \theta)}{\rho^{e}}+h-\frac{\omega^{2}}{2 \rho^{2}}\right)\left[d \rho^{2}+\frac{\rho^{2}}{4}\left(d \varphi^{2}+\sin ^{2} \varphi d \theta^{2}\right)\right]
$$

The standard procedure for the derivation of the geodesic equations, via the calculation of the corresponding Christoffel symbols, yields the following system of equations expressed with respect to time as the independent variable :

$$
\begin{aligned}
\text { (i) } 0 & =\ddot{\rho}+\frac{\dot{\rho}^{2}}{\rho}-\frac{1}{\rho}\left(\frac{2-e}{\rho^{e}} U^{*}+2 h\right) \\
\text { (ii) } 0 & =\ddot{\varphi}+2 \frac{\dot{\rho}}{\rho} \dot{\varphi}-\frac{1}{2} \sin (2 \varphi) \dot{\theta}^{2}-\frac{4}{\rho^{2+e}} U_{\varphi}^{*} \\
\text { (iii) } \quad 0 & =\ddot{\theta}+2 \frac{\dot{\rho}}{\rho} \dot{\theta}+2 \cot (\varphi) \dot{\varphi} \dot{\theta}-\frac{4}{\rho^{2+e}} \frac{1}{\sin ^{2} \varphi} U_{\theta}^{*}
\end{aligned}
$$

Note that equation (i), associated with the radial variable $\rho$ of $\bar{M}$, as a cone over the sphere $M^{*}=S^{2}$, is simply the Lagrange-Jacobi equation, cf. (13). 
Moreover, the second equation in (13) is merely a reformulation of equation (ii) and (iii), as explained in [3], Section 3.4.2. On the other hand, the dependence on $\omega$ in the above equations is only implicit, but it appears in equation (i) via substitution of the energy integral

$$
\text { (iv) } h=\bar{T}-\bar{U}=\frac{1}{2} \dot{\rho}^{2}+\frac{\rho^{2}}{8}\left(\dot{\varphi}^{2}+\sin ^{2} \varphi \dot{\theta}^{2}\right)+\frac{\omega^{2}}{2 \rho^{2}}-\frac{U^{*}}{\rho^{e}}
$$

once we have specified the value of $h$. Equation (iv) makes any of the three equations of (30) superfluous and may be replaced by (iv), as the first step of integration, with $\omega$ appearing as an integration constant.

Let us also describe another approach to derive the ODEs in (30), namely by regarding $\bar{M}$ as a simple conservative mechanical system with the Lagrange function

$$
\bar{L}=\bar{T}+\bar{U}=T+U-\frac{\omega^{2}}{\rho^{2}}=\frac{1}{2} \dot{\rho}^{2}+\frac{\rho^{2}}{8}\left(\dot{\varphi}^{2}+\sin ^{2} \varphi \dot{\theta}^{2}\right)-\frac{\omega^{2}}{2 \rho^{2}}+\frac{U^{*}}{\rho^{e}}
$$

Then, straighforward calculations of the associated Lagranges's equations

$$
\frac{d}{d t}\left(\frac{\partial \bar{L}}{\partial \dot{\rho}}\right)=\frac{\partial \bar{L}}{\partial \rho}, \quad \frac{d}{d t}\left(\frac{\partial \bar{L}}{\partial \dot{\varphi}}\right)=\frac{\partial \bar{L}}{\partial \varphi}, \quad \frac{d}{d t}\left(\frac{\partial \bar{L}}{\partial \dot{\theta}}\right)=\frac{\partial \bar{L}}{\partial \theta}
$$

yield the system (30). Similar calculations are worked out in [3], Section 3.2.

\section{The proofs of Theorem 1.1 and Theorem 1.2}

\subsection{Separation of the scaling variable}

Let $\gamma^{*}(t)=(\varphi(t), \theta(t))$ be a given time parametrized curve on the unit sphere $S^{2}$, and set $s=s(t) \geq 0$ to be its arc-length function. Then its unit tangent and positively oriented unit normal are, respectively

$$
\boldsymbol{\tau}^{*}=\frac{d \gamma^{*}}{d s}=\frac{1}{v}\left(\dot{\varphi} \frac{\partial}{\partial \varphi}+\dot{\theta} \frac{\partial}{\partial \theta}\right), \boldsymbol{\nu}^{*}=\frac{1}{v}\left(-\dot{\theta} \sin \varphi \frac{\partial}{\partial \varphi}+\dot{\varphi} \frac{1}{\sin \varphi} \frac{\partial}{\partial \theta}\right)
$$

and its speed and scalar acceleration are, respectively

$$
v=\sqrt{\dot{\varphi}^{2}+\sin ^{2} \varphi \dot{\theta}^{2}}, \quad \dot{v}=\frac{d}{d t} v=\frac{1}{v}\left[\dot{\varphi} \ddot{\varphi}+(\sin \varphi \cos \varphi) \dot{\varphi} \dot{\theta}^{2}+\sin ^{2}(\varphi) \dot{\theta} \ddot{\theta}\right]
$$

One way to calculate the geodesic curvature function $K^{*}$ is to express $\gamma^{*}$ in Euclidean coordinates as $\mathbf{x}(s)=(x(s), y(s), z(s))$ and use the formula

$$
K^{*}(s)=\mathbf{x}(s) \times \mathbf{x}^{\prime}(s) \cdot \mathbf{x}^{\prime \prime}(s)
$$

where differentiation is with respect to $s$. Then, by returning to spherical coordinates

$$
\begin{aligned}
K^{*} & =(\cos \varphi) \theta^{\prime}\left(1+\varphi^{\prime 2}\right)+\sin \varphi\left(\varphi^{\prime} \theta^{\prime \prime}-\theta^{\prime} \varphi^{\prime \prime}\right) \\
& =\frac{1}{v^{3}}\left\{(\cos \varphi) \dot{\theta}\left(v^{2}+\dot{\varphi}^{2}\right)+\sin \varphi(\dot{\varphi} \ddot{\theta}-\dot{\theta} \ddot{\varphi})\right\}
\end{aligned}
$$


Next, let us eliminate the second order terms $\ddot{\varphi}$ and $\ddot{\theta}$ in the expression (36), using equations (ii), (iii) of the system (30). This procedure yields

$$
\begin{aligned}
K^{*} v^{3} & =(\cos \varphi) \dot{\theta}\left(v^{2}+\dot{\varphi}^{2}\right)+(\sin \varphi) \dot{\varphi}\left(-\frac{2 \dot{\rho}}{\rho} \dot{\theta}-2(\cot \varphi) \dot{\varphi} \dot{\theta}+\frac{4}{\rho^{2+e}} \frac{1}{\sin ^{2} \varphi} U_{\theta}^{*}\right) \\
& -(\sin \varphi) \dot{\theta}\left(-\frac{2 \dot{\rho}}{\rho} \dot{\varphi}+\frac{1}{2} \sin (2 \varphi) \dot{\theta}^{2}+\frac{4}{\rho^{2+e}} U_{\varphi}^{*}\right) \\
& =\frac{4}{\rho^{2+e}}\left(\frac{\dot{\varphi}}{\sin \varphi} U_{\theta}^{*}-\dot{\theta} \sin \varphi U_{\varphi}^{*}\right)=\frac{4 v}{\rho^{2+e}} U_{\nu}^{*}
\end{aligned}
$$

and consequently we arrive at the formula

$$
\rho^{2+e}=\frac{4}{v^{2}} \frac{U_{\nu}^{*}}{K^{*}}=\frac{4}{v^{2}} \mathfrak{S}
$$

Note that the function $\mathfrak{S}=\mathfrak{S}\left(\gamma^{*}\right)$, called the Siegel function in 3], depends only on the intrinsic geometry of the pair $\left(\gamma^{*}, U^{*}\right)$ on the sphere.

\subsection{Intrinsic geometry of the shape curve and the gradi- ent of $U^{*}$}

In the local analysis of the moduli and the shape curve, and their interaction with the potential function $U^{*}$, we shall distinguish between two types of variables or quantities associated with a given moduli curve $\bar{\gamma}(t)=\left(\rho(t), \gamma^{*}(t)\right)$. On the one hand, the intrinsic quantities depend only on $\gamma^{*}$ as an oriented geometric (i.e. unparametrized) curve and $U^{*}$ as a function on $S^{2}$, and on the other hand, the variable quantities are defined along $\bar{\gamma}$ or $\gamma^{*}$, depending on the scaling function $\rho$ in the moduli space $\bar{M}$ or the time parametrization of the curves.

The basic intrinsic quantities are the gradient field $\nabla U^{*}$ (or its tangential and normal derivatives $\left.U_{\tau}^{*}, U_{\nu}^{*}\right)$, the orthonormal frame field $\left\{\boldsymbol{\tau}^{*}, \boldsymbol{\nu}\right\}$ along $\gamma^{*}$, and the geodesic curvature function $K^{*}$ of $\gamma^{*}$. In general, the linkage between $\gamma^{*}$ and $U^{*}$ is neatly encoded into the intrinsic function $\mathfrak{S}=U_{\nu}^{*} / K^{*}$, introduced in (37), so we shall assume $\gamma^{*}$ is not confined to a geodesic circle (in which case $\mathfrak{S}$ is undefined).

We choose a (generic) point $P_{0}$ on $\gamma^{*}$, and let $s$ be the arc-length parameter of $\gamma^{*}$ in the positive direction starting from $P_{0}$. Then the coefficients of the following power series expansions

$$
\begin{aligned}
K^{*} & =K_{0}+K_{1} s+K_{2} s^{2}+\ldots \\
U^{*} & =u_{0}+\bar{u}_{1} s+\bar{u}_{2} s^{2}+\ldots \\
U_{\tau}^{*} & =\bar{u}_{1}+2 \bar{u}_{2} s+3 \bar{u}_{3} s^{2}+\ldots \\
U_{\nu}^{*} & =\omega_{0}+\omega_{1} s+\omega_{2} s^{2}+\ldots \\
\mathfrak{S} & =\mathfrak{S}_{0}+\mathfrak{S}_{1} s+\mathfrak{S}_{2} s^{2}+\ldots
\end{aligned}
$$


yield intrinsic quantities (or geometric data) localized at the point $P_{0}$. The coefficients $\mathfrak{S}_{n}$ are expressible as rational functions of $K_{i}$ and $\omega_{i}$, and generally, let us say the order of a coefficient in (38) is the highest order of derivatives of local coordinates in its expression. Thus, we say $\varphi_{0}, \theta_{0}$ and $u_{0}$ are the intrinsic geometric data of order 0 at $P_{0}$, and for example, $\bar{u}_{1}, \omega_{0}$ and $\left.\tau^{*}\right|_{P_{0}}$ have order 1 , and $\omega_{n}, \bar{u}_{n+1}\left(\operatorname{resp} . K_{n}, \mathfrak{S}_{n}\right)$ have order $n+1$ (resp. $\left.n+2\right)$.

Let $(\varphi, \theta)$ denote spherical polar coordinates so that $P_{0}$ is different from any of the "poles" $\varphi=0$ or $\pi$. We shall expand the coordinate functions of $\bar{\gamma}(t)$, as well as $U^{*}$ and its partial derivatives, as power series with respect to $t$ :

$$
\begin{aligned}
\rho & =\rho_{0}+\rho_{1} t+\rho_{2} t^{2}+\rho_{3} t^{3}+\ldots \\
\varphi & =\varphi_{0}+\varphi_{1} t+\varphi_{2} t^{2}+\varphi_{3} t^{3}+\ldots \\
\theta & =\theta_{0}+\theta_{1} t+\theta_{2} t^{2}+\theta_{3} t^{3}+\ldots \\
v & =v_{0}+v_{1}+v_{2} t+v_{2} t^{2}+\ldots \\
U^{*} & =u_{0}+u_{1} t+u_{2} t^{2}+u_{3} t^{3}+\ldots \\
U_{\varphi}^{*} & =\mu_{0}+\mu_{1} t+\mu_{2} t^{2}+\mu_{3} t^{3}+\ldots \\
U_{\theta}^{*} & =\eta_{0}+\eta_{1} t+\eta_{2} t^{2}+\eta_{3} t^{3}+\ldots
\end{aligned}
$$

For convenience, some of the initial coefficiens are

$$
\begin{aligned}
& u_{0}=U^{*}\left(\varphi_{0}, \theta_{0}\right), \quad u_{1}=\mu_{0} \varphi_{1}+\eta_{0} \theta_{1}, \text { etc. } \\
& f_{0}=\sin \left(2 \varphi_{0}\right), f_{1}=2 \cos \left(2 \varphi_{0}\right) \varphi_{1}, \text { etc. } \\
& g_{0}=\sin ^{2}\left(\varphi_{0}\right), \quad g_{1}=f_{0} \varphi_{1}, \text { etc. } \\
& v_{1}=\frac{1}{v_{0}}\left[2 \varphi_{1} \varphi_{2}+\sin \left(\varphi_{0}\right) \cos \left(\varphi_{0}\right) \varphi_{1} \theta_{1}^{2}+2 \sin ^{2}\left(\varphi_{0}\right) \theta_{1} \theta_{2}\right]
\end{aligned}
$$

where $v_{1}$ follows from (35), and we also write

$$
\begin{aligned}
& \sin (2 \varphi)=f_{0}+f_{1} t+f_{2} t^{2}+\ldots \\
& \sin ^{2}(\varphi)=g_{0}+g_{1} t+g_{2} t^{2}+\ldots
\end{aligned}
$$

We shall regard $\mu_{0}, \eta_{0}$ as intrinsic data, but they depend on the coordinate system, of course.

Below we shall investigate dependence relations among the coefficients in (39) such as $\rho_{i}, \varphi_{j}, \theta_{k}$ and various other coefficients. Some of them are directly expressible in terms of the intrinsic data and hence regarded as constants, whereas the others are the variables.

Definition 3.1 The following list of coefficients in the expansions (39)

$$
\rho_{0}, v_{0} ; \rho_{1}, \varphi_{1}, \theta_{1} ; \rho_{2}, \varphi_{2}, \theta_{2}
$$

will be referred to as the variables of order $\leq 2$. The variables of order $n$ are $\rho_{n}, \varphi_{n}, \theta_{n}$ when $n>0$, and $\rho_{0}, v_{0}$ are the only variable of order zero. 
Henceforth, assume the above moduli curve $\bar{\gamma}(t)$ is a solution of the ODE system (30)-(31) with $e=1$. By inserting the power series into the equations (i)-(iv) and applying the method of undetermined coefficients, we arrive at the following scheme of recursive relations for the variables of increasing order $0,1,2 .$. :

$$
\begin{aligned}
& E_{10}: 0=2 \rho_{0}^{2} \rho_{2}+\rho_{0} \rho_{1}^{2}-2 h \rho_{0}-u_{0} \\
& E_{20}: 0=2 \rho_{0}^{3} \varphi_{2}+2 \rho_{0}^{2} \rho_{1} \varphi_{1}-\frac{1}{2} \rho_{0}^{3} f_{0} \theta_{1}^{2}-4 \mu_{0} \\
& E_{30}: 0=2 g_{0} \rho_{0}^{3} \theta_{2}+2 g_{0} \rho_{0}^{2} \rho_{1} \theta_{1}+\rho_{0}^{3} f_{0} \varphi_{1} \theta_{1}-4 \eta_{0} \\
& E_{40}: 0=\rho_{0}^{2} \rho_{1}^{2}+\frac{1}{4} \rho_{0}^{4}\left(\varphi_{1}^{2}+g_{0} \theta_{1}^{2}\right)+\omega^{2}-2 u_{0} \rho_{0}-2 h \rho_{0}^{2}
\end{aligned}
$$

and in general

$$
\begin{aligned}
& E_{1 n}: 0=(n+2)(n+1) \rho_{0}^{2} \rho_{n+2}+\ldots \ldots \\
& E_{2 n}: 0=(n+2)(n+1) \rho_{0}^{3} \varphi_{n+2}+\ldots . . \\
& E_{3 n}: 0=(n+2)(n+1) g_{0} \rho_{0}^{3} \theta_{n+2}+\ldots \ldots
\end{aligned}
$$

where the remaining terms are of less order since they involve $\rho_{i}, \varphi_{i}, \theta_{i}$ for $i<$ $n+2$. For example, the order of the coefficients $u_{n}, \mu_{n}, \eta_{n}$ in (43) is $n$. The equations $E_{4 n}$ for $n>0$ will not be needed and hence omitted since they do not lead to additional (algebraic independent) relations.

Now, let us select some independent and recursive relations from the above ones, but first we take the basic identity (37) and the expression (35) for the speed in the spherical metric, whose zero order terms yield the two identities :

$$
\begin{aligned}
& E_{0}: \rho_{0}^{3} v_{0}^{2}=4 \mathfrak{S}_{0} \\
& E_{0}^{\prime}: v_{0}=\sqrt{\varphi_{1}^{2}+g_{0} \theta_{1}^{2}}
\end{aligned}
$$

We shall use the symbols $J_{1}, J_{2}$ etc. to denote various expressions which are of intrinsic type. By using (44) the identities $E_{10}$ and $E_{40}$ can be restated as

$$
\begin{array}{lr}
E_{1}: \rho_{0}\left(\rho_{1}^{2}-2 h\right)+\frac{\omega^{2}}{\rho_{0}}=J_{1}, & J_{1}=2 u_{0}-\mathfrak{S}_{0} \\
E_{4}: \rho_{0}^{2} \rho_{2}-\frac{\omega^{2}}{2 \rho_{0}}=J_{4}, & J_{4}=\frac{1}{2}\left(-u_{0}+\mathfrak{S}_{0}\right)
\end{array}
$$

Next, the direction $\psi_{0}$ of $\gamma^{*}$ at the point $\left(\varphi_{0}, \theta_{0}\right)$ is intrinsic; it is also conveniently represented by the unit tangent vector

$$
\tau^{*}=\frac{1}{v_{0}}\left(\varphi_{1} \frac{\partial}{\partial \varphi}+\theta_{1} \frac{\partial}{\partial \theta}\right)=J_{\varphi} \frac{\partial}{\partial \varphi}+J_{\theta} \frac{\partial}{\partial \theta}
$$

The coefficients $J_{\varphi}, J_{\theta}$ are intrinsic functions, depending on the coordinate system, and they are related by the identity

$$
J_{\varphi}^{2}+g_{0} J_{\theta}^{2}=1
$$


Therefore, we adjoin to our list (46) the two identities

$$
\begin{aligned}
& E_{2}: \varphi_{1}=J_{\varphi} v_{0} \\
& E_{3}: \theta_{1}=J_{\theta} v_{0}
\end{aligned}
$$

Still, we have not used all zero order relations, namely $E_{20}$ and $E_{30}$, and now we state them as

$$
\begin{array}{ll}
E_{5}: \rho_{0}^{3} \varphi_{2}+\rho_{0}^{2} \rho_{1} \varphi_{1}=J_{5}, & J_{5}=2 \mu_{0}+f_{0} J_{\theta}^{2} \mathfrak{S}_{0} \\
E_{6}: \rho_{0}^{3} \theta_{2}+\rho_{0}^{2} \rho_{1} \theta_{1}=J_{6}, & J_{6}=\frac{2 \eta_{0}}{g_{0}}-2 \frac{f_{0}}{g_{0}} J_{\varphi} J_{\theta} \mathfrak{S}_{0}
\end{array}
$$

By continuing this way, we obtain for each $n>0$ three new relations with leading terms as indicated

$$
\begin{aligned}
& E_{3 n+1}: 0=\rho_{0}^{2} \rho_{n+2}+\ldots . \\
& E_{3 n+2}: 0=\rho_{0}^{3} \varphi_{n+2}+\ldots . . \\
& E_{3 n+3}: 0=\rho_{0}^{3} \theta_{n+2}+\ldots \ldots
\end{aligned}
$$

where the triple $\rho_{n+2}, \varphi_{n+2}, \theta_{n+2}$ are the variables of highest order $n+2$.

Claim 3.2 It is possible to solve the above recursive relations for the variables 41) completely in terms of the intrinsic local geometric data in the shape space.

We proceed as follows. At this point, we observe first that there are altogether $3 n+8$ variables

$$
\rho_{0}, v_{0} ; \rho_{1}, \varphi_{1}, \theta_{1} ; \rho_{2}, \varphi_{2}, \theta_{2} ; \ldots ; \rho_{n+2}, \varphi_{n+2}, \theta_{n+2} ;
$$

involved in $3 \mathrm{n}+8$ recursive relations, and the first eight involve only the variables up to order 2. However, $E_{0}^{\prime}, E_{2}$ and $E_{3}$ are obviously algebraic dependent due to the identity (47), so we shall search for one more independent relation among the variables of order $\leq 2$. We expect such a relation to involve local intrinsic quantities of order (at least) 3, so a natural approach is to differentiate the basic identity (37) involving the function $\mathfrak{S}$. Then, evaluation of the resulting identity at $t=t_{0}$ yields

$$
3 \frac{\rho_{1}}{\rho_{0} v_{0}}+2 \frac{v_{1}}{v_{0}^{2}}=J_{7}, \quad J_{7}=\frac{\mathfrak{S}_{1}}{\mathfrak{S}_{0}}
$$

Using the expression in (40) for $v_{1}$ we can restate the above identity as

$$
3 \frac{\rho_{1}}{\rho_{0} v_{0}}+\frac{4}{v_{0}^{3}}\left[\varphi_{1} \varphi_{2}+\frac{1}{4} f_{0} \varphi_{1} \theta_{1}^{2}+g_{0} \theta_{1} \theta_{2}\right]=J_{7}
$$


By simple calculation and substitution using some of the previous relations $E_{i}$,

$$
\begin{aligned}
& \rho_{0}^{3}\left[\varphi_{1} \varphi_{2}+\frac{1}{4} f_{0} \varphi_{1} \theta_{1}^{2}+g_{0} \theta_{1} \theta_{2}\right] \\
& =\varphi_{1}\left(J_{5}-\rho_{0}^{2} \rho_{1} \varphi_{1}\right)+\varphi_{1}\left(\frac{1}{4} f_{0} J_{\theta}^{2} \rho_{0}^{3} v_{0}^{2}\right)+\theta_{1}\left(g_{0} J_{6}-g_{0} \rho_{0}^{2} \rho_{1} \theta_{1}\right) \\
& =-\frac{\rho_{1}}{\rho_{0}} \rho_{0}^{3}\left(\varphi_{1}^{2}+g_{0} \theta_{1}^{2}\right)+\varphi_{1}\left(J_{5}+f_{0} J_{\theta}^{2} \mathfrak{S}_{0}\right)+\theta_{1} g_{0} J_{6} \\
& =-4 \frac{\rho_{1}}{\rho_{0}} \mathfrak{S}_{0}+v_{0}\left[J_{\varphi} J_{5}+f_{0} J_{\varphi} J_{\theta}^{2} \mathfrak{S}_{0}+g_{0} J_{\theta} J_{6}\right]
\end{aligned}
$$

and by substitution into (52), using the identity $\rho_{0}^{3} v_{0}^{2}=4 \mathfrak{S}_{0}$ and the expressions for $J_{5}, J_{6}$ in (49), this leads to our new identity

$$
E_{1}^{\prime}: \frac{\rho_{1}}{\rho_{0} v_{0}}=J_{8}, \quad J_{8}=2 \mathfrak{S}_{0}^{-1}\left(J_{\varphi} \mu_{0}+J_{\theta} \eta_{0}\right)-J_{7}=\frac{1}{\mathfrak{S}_{0}}\left(2 \bar{u}_{1}-\mathfrak{S}_{1}\right)
$$

where $\bar{u}_{1}$ is the tangential derivative $U_{\tau}^{*}$ of $U^{*}$ at $P_{0}$, cf. (38).

From the system of algebraic equations

$$
E_{0}, E_{0}^{\prime}, E_{1}, E_{1}^{\prime}, E_{2}, E_{3}, E_{4}, \ldots
$$

we can now solve recursively and thus determine the variables

$$
\rho_{0}, v_{0}, \rho_{1}, \varphi_{1}, \theta_{1}, \rho_{2}, \varphi_{2}, \theta_{2}, \ldots \ldots
$$

successively in terms of the intrinsic data. In fact, this is obvious from the structure of the equations, once we have determined $\rho_{0}, v_{0}, \rho_{1}$, namely using the three equations $E_{0}, E_{1}, E_{1}^{\prime}$ :

$$
\rho_{0}^{3} v_{0}^{2}=4 \mathfrak{S}_{0}, \quad \rho_{0}\left(\rho_{1}^{2}-2 h\right)+\frac{\omega^{2}}{\rho_{0}}=J_{1}, \quad \frac{\rho_{1}}{\rho_{0} v_{0}}=J_{8}
$$

It follows that $\rho_{0}$ is characterized as a positive root of the following polynomial of order $\leq 2$ :

$$
2 h \rho_{0}^{2}-\left(4 J_{8}^{2} \mathfrak{S}_{0}-J_{1}\right) \rho_{0}-\omega^{2}=0
$$

The case $\omega=0$ is discussed in 3 , Section 4.1 , and we recall the three cases $h=0, h>0, h<0$ are characterized by the sign of $4 J_{8}^{2} \mathfrak{S}_{0}-J_{1}$, namely the cases are

$$
u_{0}=\frac{1}{2}\left(4 J_{8}^{2}+1\right) \mathfrak{S}_{0}, \quad u_{0}<\frac{1}{2}\left(4 J_{8}^{2}+1\right) \mathfrak{S}_{0}, \quad u_{0}>\frac{1}{2}\left(4 J_{8}^{2}+1\right) \mathfrak{S}_{0}
$$

Now, assume $\omega>0$. In the case of $h=0$ we have clearly

$$
\rho_{0}=\frac{\omega^{2}}{J_{1}-4 J_{8}^{2} \mathfrak{S}_{0}} \quad, \quad u_{0}>\frac{1}{2}\left(4 J_{8}^{2}+1\right) \mathfrak{S}_{0}
$$


For $h>0$ the solution must be

$$
\rho_{0}=\frac{1}{4 h}\left(\left(4 J_{8}^{2} \mathfrak{S}_{0}-J_{1}\right)+\sqrt{\left(4 J_{8}^{2} \mathfrak{S}_{0}-J_{1}\right)^{2}+8 h \omega^{2}}\right)
$$

but as a further characterization we cannot rule out any of the three types of constraints (56).

In the third case, $h<0$, the two roots of equation (55) would be negative if $4 J_{8}^{2} \mathfrak{S}_{0}-J_{1}>0$, so the inequality " $\leq$ " must hold and consequenly

$$
\begin{gathered}
\rho_{0}=\frac{1}{4 h}\left(\left(4 J_{8}^{2} \mathfrak{S}_{0}-J_{1}\right)-\sqrt{\left(4 J_{8}^{2} \mathfrak{S}_{0}-J_{1}\right)^{2}+8 h \omega^{2}}\right) \\
u_{0}-\frac{1}{2}\left(4 J_{8}^{2}+1\right) \mathfrak{S}_{0} \geq \omega \sqrt{2|h|}>0
\end{gathered}
$$

The other choice of root in the formula for $\rho_{0}$ is ruled out by demanding continuous dependence on the parameters, e.g. $\omega \rightarrow 0$ should not imply $\rho_{0} \rightarrow 0$.

Finally, with the above value for $\rho_{0}$, the system (56) yields the following intrinsic formulae for the two variables $v_{0}, \rho_{1}$, namely

$$
v_{0}=2 \sqrt{\frac{\mathfrak{S}_{0}}{\rho_{0}^{3}}}, \quad \rho_{1}=2 J_{8} \sqrt{\frac{\mathfrak{S}_{0}}{\rho_{0}}}
$$

\subsection{Summary and final proofs}

To complete the proofs of Theorem 1.1 and Theorem 1.2 let us start with a curve $\bar{\gamma}(t)=(\rho(t), \varphi(t), \theta(t))$ in $\bar{M}$ which is the moduli curve of a trajectory $\gamma(t)$ of a planary 3-body motion. Then the curve $\gamma(t)$ in $M$ is uniquely determined, up to a global congruence, by the curve $\bar{\gamma}(t)$ and the size $|\boldsymbol{\Omega}|$ of the angular momentum vector. We refer to (2], Theorem B) for the purely kinematic result concerning the general lifting of curves $\bar{\gamma}(t)$ in $\bar{M}$ to curves in $M$. On the other hand, by the formula (37), the size function $\rho(t)$ is already determined by the shape curve $\gamma^{*}(t)=(\varphi(t), \theta(t))\left(\gamma^{*}\right.$ assumed to be non-exceptional $)$, and this proves Theorem 1.1

In Section 3.2 it is demonstrated that the power series expansion of $\gamma^{*}(t)$ is essentially determined by quantities which depend only on the geometric (i.e. unparametrized) shape curve $\gamma^{*}$ and the relative geometry between $\gamma^{*}$ and the gradient vector field $\nabla U^{*}$. By "essential" we mean that the same shape curve can only be reparametrized in the trivial way, namely by an affine transformation of time, in order to remain the time parametrized shape curve of a (planary) 3-body motion. In view of Remark 1.3 this completes the proof of Theorem 1.2

\section{References}

[1] W.Y. Hsiang and E. Straume, Kinematic geometry of triangles with given mass distribution, PAM-636 (1995), Univ. of Calif., Berkeley. 
[2] W.Y. Hsiang and E. Straume, Kinematic geometry of triangles and the study of the three-body problem, a memoir at NTNU (2005), arXiv: math-ph/0608060

[3] W.Y. Hsiang and E. Straume, Global geometry of 3-body motions with vanishing angular momentum,I, PAM-805 (2006), Univ. of Calif., Berkeley, arXiv: math-ph/0609076

[4] C.G.J. Jacobi, Vorlesungen über Dynamik, Berlin (1866), A. Clebsch ed.

[5] J.L. Lagrange, Essai sur le problème de trois corps, Ouvres, vol. 6 (1772), 229-324.

[6] C.L. Siegel, Lectures on the Singularities of the Three-body Problem, Tata Institute of Fundamental Research, Lectures on Mathematics, no. 42.

[7] C.L. Siegel and J. Moser, Lectures on Celestial Mathematics, Die Grundlehren der Mathematischen Wissenschaften, vol. 187, Springer-Verlag 1971. 